\title{
New experiments to validate the radiation pattern of the Middle Atmosphere Alomar Radar System (MAARSY)
}

\author{
T. Renkwitz ${ }^{1}$, G. Stober ${ }^{1}$, R. Latteck ${ }^{1}$, W. Singer ${ }^{1}$, and M. Rapp ${ }^{2}$ \\ ${ }^{1}$ Leibniz-Institute of Atmospheric Physics at the Rostock University, Schloss-Str. 6, 18225 Kühlungsborn, Germany \\ ${ }^{2}$ German Aerospace Center, Institute of Atmospheric Physics, Münchner Straße 20, \\ 82234 Oberpfaffenhofen-Wessling, Germany
}

Correspondence to: T. Renkwitz (renkwitz@iap-kborn.de)

\begin{abstract}
The Middle Atmosphere Alomar Radar System (MAARSY) is a monostatic radar with an active phased array antenna designed for studies of phenomena in the mesosphere and lower thermosphere. Its design in particular the flexible beam forming and steering capability makes it to a powerful instrument to perform observations with high angular and temporal resolution. The knowledge of the actual radiation pattern is crucial to configure and analyze experiments carried out with the radar. The simulated radiation pattern is evaluated by the observation of cosmic radio emissions which are compared to a Global Sky temperature Maps model consisting of the most recent, thorough and accurate radio astronomy surveys. Additionally to these passive receive-only experiments active two-way experiments are presented, which corroborate the findings of the passive experiments.
\end{abstract}

\section{Introduction}

In this paper we present the recent experiments performed to validate the simulated radiation pattern of MAARSY. The Middle Atmosphere Alomar Radar System was built in 2009/2010 by the Leibniz-Institute of Atmospheric Physics (IAP) on the island Andøya in Northern Norway $\left(69.30^{\circ} \mathrm{N}, 16.04^{\circ} \mathrm{E}\right)$. MAARSY allows improved studies of the arctic atmosphere in the troposphere/lower stratosphere and the mesosphere/lower thermosphere with high spatial and temporal resolution. The monostatic radar operates at $53.5 \mathrm{MHz}$ with an active phased array antenna consisting of 433 Yagi antennas. Each individual antenna is connected to its own transceiver with independent phase and amplitude control. These properties give the radar a very high flexibility of beam forming and beam steering. A detailed description of the radar is given by Latteck et al. (2012). During the design phase of MAARSY and the successive commissioning several model studies have been carried out in order to estimate the radiation pattern for various combinations of beam forming and steering. However, the actual radiation pattern may be inflicted by parameters like mutual coupling, active impedances and ground parameters. These possible impacts can hardly be measured or included into the model, which requires experimental methods to verify the model results. For this purpose, the radar has occasionally been used in passive mode, monitoring the noise power originating from both distinct galactic radio sources like e.g. Cassiopeia A (RA: $23 \mathrm{~h} 23.4 \mathrm{~m}$, Dec: $+58^{\circ} 48.9^{\prime}$ ), Cygnus A (RA: $19 \mathrm{~h} 59.473 \mathrm{~m}$, Dec: $+40^{\circ} 44.035^{\prime}$ ) and the complete diffuse galactic background noise. The analysis of this receive-only mode data enables us to verify beam forming and steering attempts. These results document the current status of the radar and provide valuable information for further improvement. Similar attempts were described in e.g. Czechowsky et al. (1984), Palmer et al. (1996), Zecha (1999), Campistron et al. (2001), Carey-Smith et al. (2003), Fukao et al. (2003), Swarnalingam et al. (2009a), Swarnalingam et al. (2009b), Renkwitz et al. (2011).

In the subsequent sections we will describe and analyze the experiments we used to evaluate the current radiation pattern of the MAARSY radar. First we will focus on passive experiments monitoring galactic radio emissions and compare the observations to a model of Global Sky temperature Maps of radio emissions (GSM). Subsequently we will present active experiments where we used satellites and Earth's moon as a target. Finally we will present a summary and conclusions for the current stage of this study and give an outlook to forthcoming experiments. 


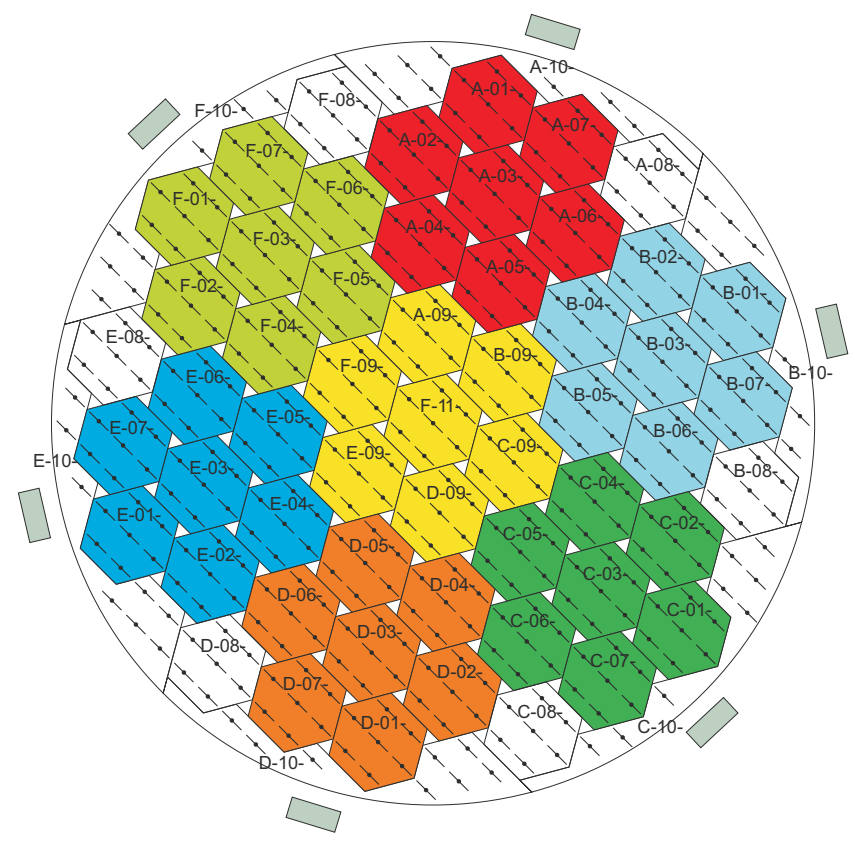

Fig. 1. Sketch of the MAARSY VHF radar antenna array. The colored subgroups mark the MAARSY343 subarray.

\section{Passive experiments observing cosmic radio noise with MAARSY}

Earlier experiments observing cosmic radio emissions with MAARSY were limited to the use of almost $80 \%$ of the MAARSY antenna array, named MAARSY343 (see, Renkwitz et al., 2012). The amount of antennas was limited by the constraint of selectable antenna groups that may be connected to the 16 receiving channels (Latteck et al., 2012). To generate the total radar beam the individual receiving signals from the antenna groups have to be coherently integrated. For these experiments we used seven antenna groups, each composed of 49 antennas, resulting in 343 individual antennas in total. With this arrangement we were able to identify three distinct cosmic radio sources, Cassiopeia A, Cygnus A and Taurus A (RA $05 \mathrm{~h} 34 \mathrm{~m}$ 31.97 s, Dec. $+2200^{\prime} 52.1^{\prime \prime}$, studied by e.g. Vinyajkin, 2005), and the signature of the Milky Way. For the analysis of beam forming and pointing and the estimation of the beam width we concentrated on the two major natural radio sources besides the sun - that can be observed in the lower VHF band on the northern hemisphere. As Taurus A is positioned at only $22^{\circ}$ declination, it is generally out of the beam pointing cone of MAARSY. However, the observation of this radio source has already been used to demonstrate the capability to steer the radar beam to such far off-vertical directions.

With the observation of galactic radio emissions we focus on the angular and temporal power distribution originating from Supernova remnants, radio galaxies and the diffuse cosmic background radiation. In the experiments we performed a scan between 0 and $34^{\circ}$ zenith angle pointing to $180^{\circ}$ in azimuth. These experiments were scheduled with other experiments in sequence for a total observation time of few days. The subsequent comparison of our observations with the Global Sky temperature Maps (GSM) by de Oliveira-Costa et al. (2008) revealed the high similarity between both. This model is comprised of the eleven most accurate sky surveys and allows the generation of Quiet Day Curves for a specific frequency chosen by the user. To adapt the native resolution of the GSM to the radar we convolved the GSM data with the simulated radiation pattern and included the typical noise figure of the receiver front ends and the losses in the coaxial cable to the antennas. Finally, we found a very good agreement between the GSM data and our observations pointed out by a correlation factor of 0.98 . As the total detected incident power is depending on the comprehensive radiation pattern including all side lobes, we were confident that the radiation pattern is similar to what has been simulated afore. For more details of the experiment settings and analysis see Renkwitz et al. (2012) and references therein.

To underline these findings and to confirm the apparent impact of the side lobes, we performed another experiment using the total antenna array for a campaign based cosmic radio noise observation. For this purpose all groups of the antenna array were connected to individual receiver channels. The results of this experiment have been presented in Renkwitz et al. (2013). We clearly found an increase of incident power for the distinct radio sources Cassiopeia A and Cygnus A and a decrease of power around the "cold" part of the sky resulting in approximately $1 \mathrm{~dB}$ more dynamic of incident power. This confirmed the obvious assumption of the radiation patterns impact to the detected galactic radio emissions. In the most recent sky noise experiments we were able to use both, a hardware combined beam of the total antenna array (MAARSY433) and the earlier described MAARSY343 simultaneously. The simulated radiation pattern of MAARSY433 and MAARSY343 antenna array with an overlay of the trajectories of Cassiopeia A and Cygnus A are depicted in figure 2.

\section{Comparison of observations with a Global Sky temperature Maps model}

In this section we will compare the observations of galactic radio emissions done with MAARSY to the Global Sky temperature Maps (GSM) of de Oliveira-Costa et al. (2008). One of the first extensive surveys has been presented by Haslam et al. (1974), Haslam et al. (1981) and Haslam et al. (1982) for a frequency of $408 \mathrm{MHz}$, which is still used as an all sky reference nowadays. Detailed studies of cosmic radio emissions at approximately $1.4 \mathrm{GHz}$ were presented by e.g. Reich et al. (1997). For lower frequencies the earlier cosmic sky noise surveys e.g. Cane (1978) are 

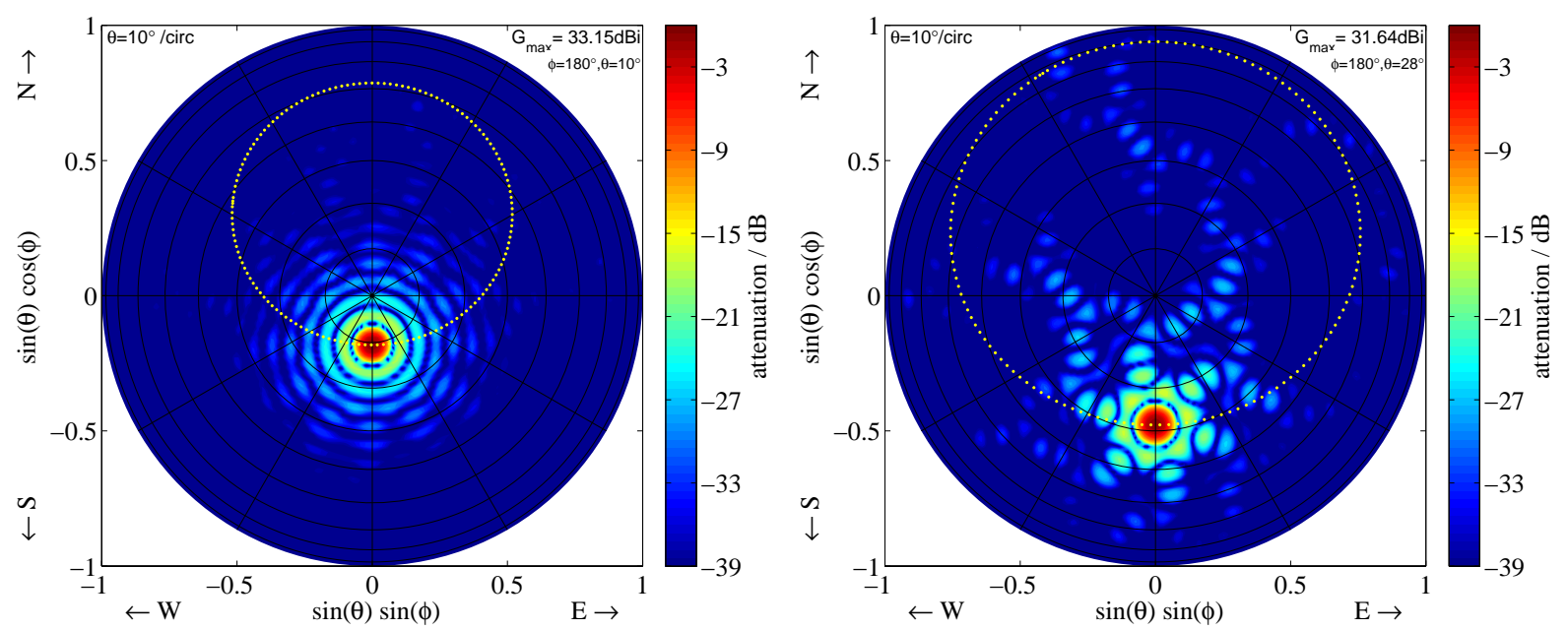

Fig. 2. Simulated radiation pattern of the MAARSY antenna array in top view, using 433 array elements while pointing to the radio sources Cassiopeia A $\left(\phi=180^{\circ}, \theta=10.5^{\circ}\right.$ - left side) and 343 array elements pointing to Cygnus $\mathrm{A}\left(\phi=180^{\circ}, \theta=27.5^{\circ}-\right.$ right side). The corresponding trajectories of both radio sources are depicted with dotted circles. The individual radiation pattern are depicted relative to the maximum gain with an overlay of equidistant rings of each $10^{\circ}$ zenith angle. For these simulations the Numerical Electromagnetics Code (NEC) with the Norton-Sommerfeld approximation was used implicating the mutual coupling of antennas and the influence of imperfect ground.

based on observations with a $11^{\circ}$ wide beam. The map of this survey appears smoothed due to the larger beam width in respect of MAARSY, thus, it does not show the dynamic range. However, there have been narrow beam studies at $22 \mathrm{MHz}$ by Roger et al. (1999). Every of these studies implies valuable information for further comparisons, while the difference in the observation frequency has to be considered. Recently, de Oliveira-Costa et al. (2008) derived a model to create global sky temperature maps for any user defined frequency in the range from $10 \mathrm{MHz}$ to $100 \mathrm{GHz}$. The model is based on the merging of the eleven most accurate radio astronomy surveys. At our frequency of interest, this results in a GSM with significantly increased resolution compared to other sources. The accuracy of the GSM is given to $1-10 \%$ depending on frequency and sky region. For our observation frequency the GSM is highly dependent on the $45 \mathrm{MHz}$ surveys of Maeda et al. (1999), which have been performed with the MU radar for the Northern declinations and on Roger et al. (1999) using the $22 \mathrm{MHz}$ DRAO radio telescope. The angular resolution and beam width in especially the latter survey is better than the beam width we used for the observations with MAARSY. The fine structure in the GSM relies on observations performed on higher frequencies. Hence the model data offers an appropriate angular resolution for further comparisons. A similar approach have been used by Stober et al. (2011) for the calibration of meteor radars.

In Renkwitz et al. (2012) we concentrated on the comparison of quiet day curves (QDC) obtained by the radar observations and the GSM data. These QDC were generated for fixed zenith angles for an entire sidereal day.
Recently, we pursued this comparison by creating 2-D GSM maps matching to the scan experiments we performed with MAARSY.

We generated these GSM maps with a resolution of $2^{\circ}$, besides the apparent zenith pointing angle to the major noise sources Cassiopeia A and Cygnus A. However, we possibly not exactly matched the position of these sources in the GSM. Subsequently, the GSM map was interpolated to a resolution of $1^{\circ}$, which is the typical angular step size of the observations. To adapt MAARSY to the GSM data, we needed to include a polarization efficiency factor since in 2012 the upgrade of MAARSY to circular polarization has been initiated. Hence, one hexagon and one anemone group, with 7 and 49 antennas respectively, were converted to circular polarization. The polarization efficiency is estimated strictly to the amount of circular polarized antennas to the total antenna array used in the individual experiments and the loss in the polarization unit and feeding system, leading to 0.52 and 0.525 for MAARSY433 and MAARSY343 respectively. Furthermore, the current status of the ongoing polarization upgrade of MAARSY inflicts the apparent radiation pattern, where the side lobes are slightly deteriorated due to amplitude tapering. It has to be noted that for the generation of the GSM maps the ideal simulated radiation patterns have been used, disregarding the actual amplitude tapering. Therefore we assume to see more dynamic range of the incident power in the GSM maps than in the observations. Chau et al. (2013) presented a method to gain information about the actual phase distribution of the antenna groups and the dedicated receivers. The derived phases have been considered to 

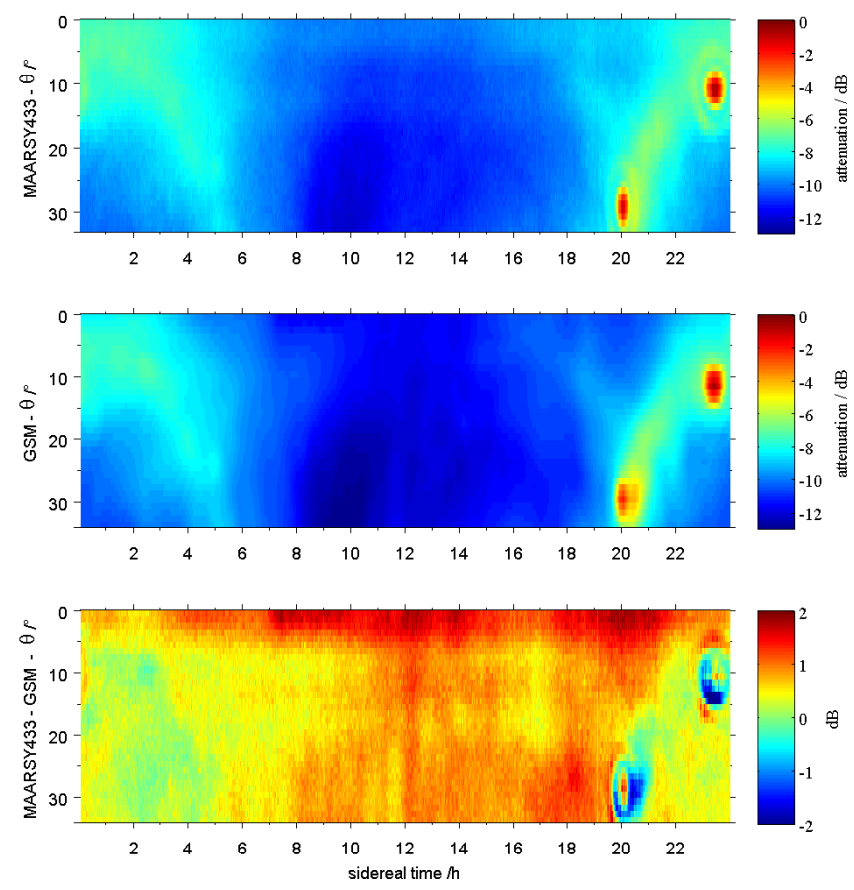

Fig. 3. Distribution of incident noise power over local sidereal time and zenith angle $\theta$ for a scan within $\phi=180^{\circ}, \theta=0-34^{\circ}$ received with MAARSY433. The highlighted point-like radio sources are Cassiopeia A $\left(\theta=10.5^{\circ}\right)$ and Cygnus A $\left(\theta=27.5^{\circ}\right)$. The middle and bottom panel show the generated GSM map for the equivalent scan and the deviation from the observation.

generate the MAARSY343 beam which is used in the recent experiments, presented in this paper. Figures 3 and 4 show the detected incident power in the upper panel, succeeded by the GSM map generated for the associated radiation pattern and finally the apparent deviation for both MAARSY433 and MAARSY343 respectively. It has to be noted that the observation data comprises a single polar winter day in end of November 2012 but characterized by very low geomagnetic activity and distortions related to interference. In general we found a very good agreement to the GSM, however the individual "point-sources" Cassiopeia A and Cygnus A appear to be too broad in the GSM. Hence, we tend to rely on the widely spread incident power originating from the Milky Way and the diffuse background radiation than the accurate measurement of the distinct radio sources for both, our measurements and the surveys implicated in the GSM. For the parts of the sky representing lower temperatures we see the largest discrepancy between the GSM map and our observation. This is, to a certain degree, related to the ideal pattern used to generate the GSM maps. However, we typically see an offset of $1-1.5 \mathrm{~dB}$ in both comparisons. Additionally, the maximum intensity of Cassiopeia A seems to be shifted in the GSM map by approximately $1^{\circ}$. The dynamic of incident cosmic radio emissions within the actual experiments using MAARSY433 and MAARSY343
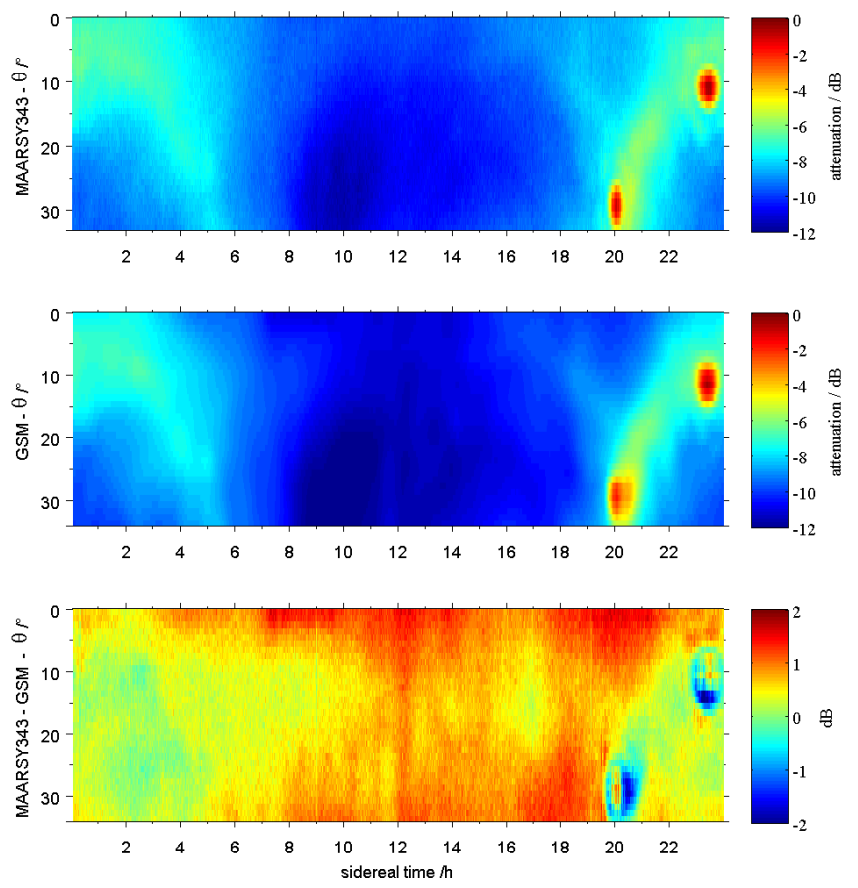

Fig. 4. Alike figure 3, distribution of incident noise power for MAARSY343, GSM map and deviation.

differs by about $0.6 \mathrm{~dB}$, while the simulated gain of the two different sets of arrays differ by about $1 \mathrm{~dB}$. However, the apparently different attenuation of side lobes in the radiation pattern and the inherently existing losses and phase deviations in the additional combiners used to generate MAARSY433 have to be considered. Furthermore, there can be seen a weak contribution of persistent noise power for zenith angles around $3^{\circ}$ and $12^{\circ}$ for MAARSY433 and around $5^{\circ}$ for MAARSY343. This practically permanent contamination seems to be related to the floodlights placed at the circumference of the antenna array, which were used for maintenance at the radar while these experiments were conducted.

Interestingly, having a detailed look into the halo around the distinct radio sources, preferably around Cassiopeia A one can identify the actual shape of the first side lobes. For the MAARSY433 array the halo appears to be round, while for MAARSY343 the halo has a hexagon shape, which is in agreement to the simulated radiation pattern. The discontinuity of the halo, south-east of Cassiopeia A and north-west of Cygnus A, appears due to the depart from the distinct radio source and the Milky Way. It has to be taken into account that the polarisation of the distinct cosmic radio sources Cassiopeia A, Cygnus A, the Milky way and the diffuse background radiation differ. This introduces variations in the detected noise power, when observing them with a fixed linear polarized antenna array (see e.g. Baars et al., 1964, Boland et al., 1966, Mayer and Hollinger, 1968, Downs and Thomson, 1972). 


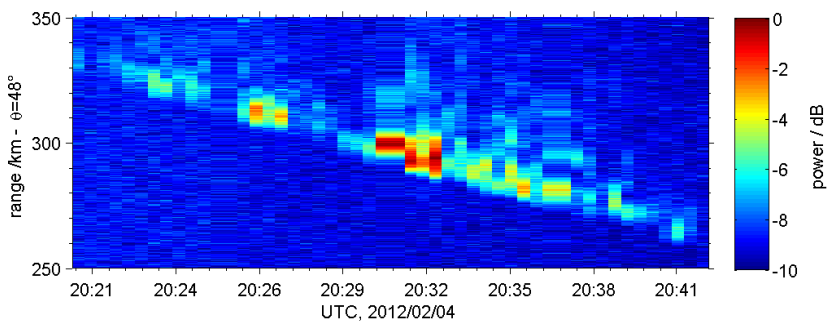

Fig. 5. Incident backscatter power received during the moon experiment depicted over range and time.

\section{Two-way active experiments}

In the beforehand presented experiment the radar was used exclusively in reception mode, while in this section we will introduce new attempts to validate the radiation pattern of MAARSY including also the transmit pattern. For this purpose we arranged experiments where we used satellites and the Earth's moon as backscatter targets.

\subsection{Moon experiment}

The limitation of the earlier presented experiments is that they only provide information about the receiving system. To gain information about the transmitting part of the radar we prepared an experiment where we actively used the radar tracking the moon. During this experiment we used the full antenna array to transmit a $50 \mu$ s long pulse equivalent to $7.2 \mathrm{~km}$ range, while for reception we used MAARSY343. Similarly to the beforehand described cosmic noise experiments we performed a scan in zenithal direction while pointing southwards to see the passage of the moon. Since the moon is generally not reaching an elevation of more than $41^{\circ}$, due to its low declination, within the next years at the polar position of MAARSY, grating lobes are generated while pointing the radar beam at such zenith angle. However these grating lobes generated at about $\phi=335^{\circ}$ and $\theta=70^{\circ}$ will likely not inflict with other targets, besides picking up additional noise for the receiver. Potential targets like satellites may be rejected due to their apparent approach speed.

A backscatter signal of the moon was found at the predicted time and zenith angle as shown in Fig. 5. Confirmation was given by analyzing the slope of this echo, what resulted in an approach speed of approximately $205 \mathrm{~km} \mathrm{~h}^{-1}$, while the simulation of the moon's speed showed $202 \mathrm{~km} \mathrm{~h}^{-1}$. The echo depicted in the figure 5 is characterized by high variations in power which may be related to fast Faraday rotation and absorption effects as the radar signal travels along a considerable long path through the mesosphere and ionosphere, pointing $48^{\circ}$ off-zenith. At the time of this experiment the geomagnetic indices showed disturbances and the surrounding magnetometer indicated variations of $\pm 200 \mathrm{nT}$ of the magnetic field components
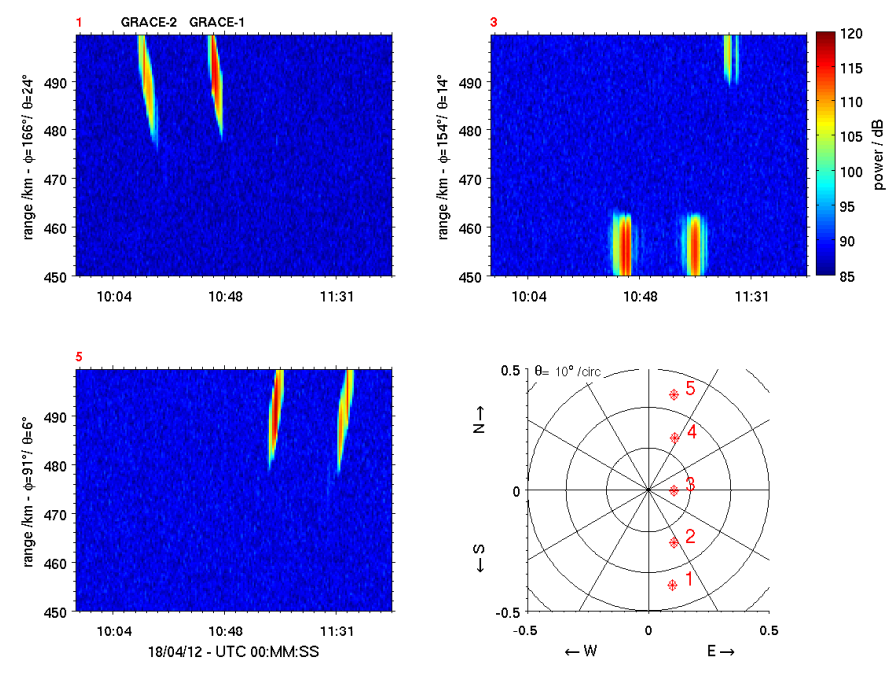

Fig. 6. Incident backscatter from the GRACE-satellite experiment for three of five beam positions depicted over range and time. The beam positions used in this experiment are shown in the lower right panel, depicted in spherical coordinates.

(see, Tromsø Geophysical Observatory (2012). Considering the beforehand limitations we could also estimate the beam pointing error within this experiment to be in the order of less than $2^{\circ}$ in azimuth and zenith. These findings are in agreement to the experiments observing the radio source Taurus A, which is connected to the Crab nebula and was successfully detected at about the same zenith angle (Renkwitz et al., 2012). Due to these passive and active experiments conducted at these high off-zenith angles, we assume similar behavior of the radiation pattern for both reception and transmission.

\subsection{Satellite experiments}

To gather information about MAARSYs beam pointing, the earlier described moon experiment was performed with the knowledge of the generation of grating lobes while pointing up to $50^{\circ}$ off-vertical. To obtain further information about the beam forming and positioning without the generation of grating lobes targets have to be chosen which are within the normal beam pointing cone of MAARSY with less than $30^{\circ}$ zenith angle. Chau et al. (2013) described a method to analyze meteor head-echoes to gain information about the main and the first side lobe of MAARSYs radiation pattern contributing to the estimation of the radiation pattern. Another way to verify the beam pointing capability of a radar is the use of satellites. We have chosen the GRACE and ENVISAT satellites as these have high inclination orbits and represent a significantly large target due to their dimensions of $3.1 \times 1.9 \mathrm{~m}$ and $26 \times 10 \mathrm{~m}$ respectively. In Fig. 6 are depicted the height-time-intensity plots for one passage of the two GRACE satellites almost over head of MAARSY. In total, five beam directions with five to $25^{\circ}$ zenith angle were 

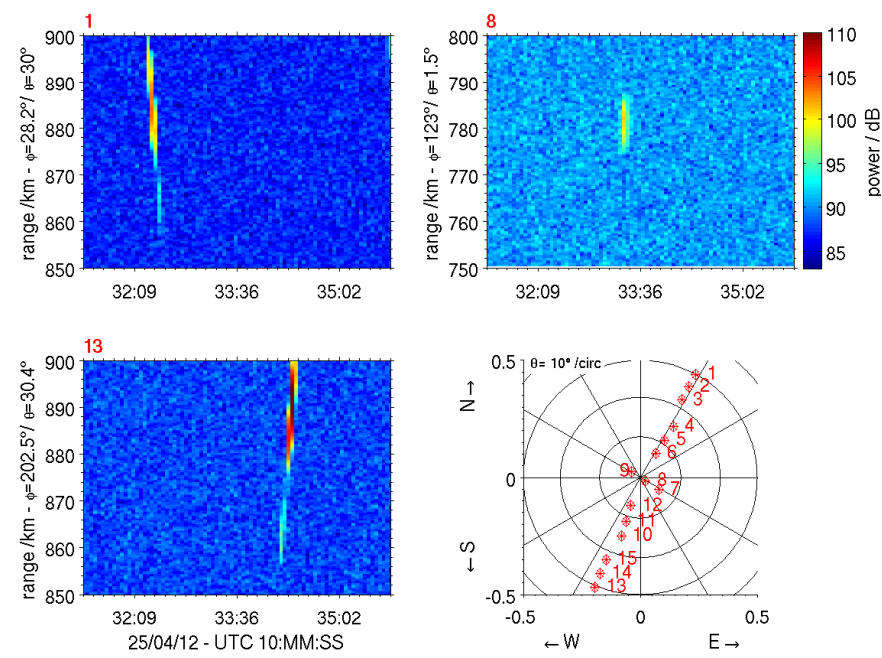

Fig. 7. Incident backscatter from the ENVISAT-satellite experiment for three of 15 beam positions depicted over range and time. The beam positions used in this experiment are shown in the lower right panel, depicted in spherical coordinates.

used and sampled for the range of $445 \mathrm{~km}$ to $500 \mathrm{~km}$, where the satellites were finally easily detected. Due to the chosen pulse length of approximately $7 \mathrm{~km}$ strong backscatter was received. The time of passage through the individual radar beams and the detected range are in good agreement to simulations of the trajectory. Subsequently, we aimed for the detection of ENVISAT, which is one of the largest targets passing MAARSYs coverage. In this experiment we used 15 radar beam positions, where 13 were pointing directly to the trajectory of ENVISAT and two beams are displaced by $4^{\circ}$. Doing so, we might get an information about the side lobe attenuation. In Fig. 7 are depicted three examples of the passage of ENVISAT through MAARSYs radar beam. Alike the experiment tracking the GRACE satellites, we received backscatter from ENVISAT in all 13 beam positions that were on the trajectory. However, we couldn't find any signal in the radar beams marked with no. 7 and 9, where both are seperated by $4^{\circ}$ in zenith angle from beam no. 8. As we detected the satellites signal in position 8 with a SNR of $10 \mathrm{~dB}$, we may state the apparent side lobe attenuation was greater than $20 \mathrm{~dB}$. Unfortunately we don't know whether the radar beam direction was exactly matching the current satellite position, since ENVISAT is out of control since the 8th of April 2012 (see European Space Agency, 2012). However it appeared to be still on the predicted orbit, which is also proven by our observation performed four days after the loss of communication with the satellite. The variation of backscatter seen in our data may be a subject to the unpredictable orientation of the spacecraft. Furthermore we have found plenty of other backscatter signals that are related to other spacecrafts, but which could not be found in a quick research on publicly available catalogues. However, some of these signals also may have been received via side lobes from explicitly large targets, which complicates the assignment. With these two satellite experiments we were able to verify our beam pointing on 18 individual positions for up to $30^{\circ}$ zenith angle. This is especially valuable as due to the polar position of MAARSY the observation of galactic radio emissions is generally restricted to southern directions.

\section{Summary and conclusions}

The simulated radiation pattern of the MAARSY antenna was evaluated by using observations of cosmic radio emissions and its comparison to a Global Sky temperature Map consisting of the most recent and accurate radio astronomy surveys. Using different amounts of array elements we were able to verify the impacts of the apparent radiation pattern to the detection of cosmic radio emissions for a declination scan range of $35^{\circ}$. By the use of the entire antenna array an increase of the dynamic of nearly $1 \mathrm{~dB}$ incident cosmic radio emissions was observed. The maximum deviations between the observation and the temperature maps are in the order of up to $1.5 \mathrm{~dB}$ and are likely mainly caused by the imperfection of the presupposed simulated radiation pattern.

Additionally to these passive receive-only experiments active two-way experiments using backscatter from the moon and satellites were conducted corroborating the findings of the passive experiments. We successfully detected backscatter from satellites, verifying 18 different radar beam direction, and were additionally able to derive the moons approach speed with an apparent error of approximately $3 \mathrm{~km} \mathrm{~h}^{-1}$.

Future activities will be directed to active experiments in order to gain more information about the beam shape and the direction and attenuation of side lobes. Additionally, it is planned to use an airborne electromagnetic field probe which shall be used to directly sample the radiation pattern of MAARSY.

Acknowledgements. We like to express our gratitude to the Andøya Rocket Range for their permanent support while building and operating the MAARSY radar. Furthermore, we highly appreciate the work of de Oliveira-Costa et al. (2008), which revealed to be a very valuable tool to perform passive radar calibrations. The radar development was supported by grant 01 LP 0802A of Bundesmisterium für Bildung und Forschung. 


\section{References}

Baars, J. W. M., Mezger, P. G., and Wendker, H.: The flux density values of standard sources used for antenna calibrations, Tech. rep., National Radio Astronomy Observatory, 1964.

Boland, J. W., Hollinger, J. P., Mayer, C. H., and McCullough, T. P.: Polarization of Cygnus A, Taurus A, and Cassiopeia A at 2.07 Centimeters, Astrophys. J., 144, 437-439, 1966.

Campistron, B., Despausx, G., Lothon, M., Klaus, V., Pointin, Y., and Mauprivez, M.: A partial $45 \mathrm{MHz}$ sky temperature map obtained from the observations of five ST radars, Ann. Geophys., 19, 863-871, 2001, http://www.ann-geophys.net/19/863/2001/.

Cane, H.: A $30 \mathrm{MHz}$ map of the whole sky, Australian Journal of Physics, 31, 561-565, 1978.

Carey-Smith, T. K., McDonald, A. J., Baggaley, W. J., Bennett, R. G., Fraser, G. J., and Plank, G. E.: Antenna beam verification using cosmic noise, Proceedings of MST10 symposium, 2003.

Chau, J., Renkwitz, T., Stober, G., and Latteck, R.: MAARSY multiple receiver phase calibration using radio sources, J. Atmos. Sol.-Terr. Phy., accepted, 2013.

Czechowsky, P., Schmidt, G., and Rüster, R.: The mobile SOUSY Doppler radar: Technical design and first results, Radio Sci., 19, 441-450, 1984.

Downs, G. S. and Thomson, A. R.: The distribution of linear polarization in Cassiopeia A at wavelengths of 9.8 and $11.1 \mathrm{~cm}$, The Astronomical Journal, 77, 120-133, 1972.

European Space Agency: ENVISAT mission website, ESA EO Missions, https://earth.esa.int/web/guest/missions/ esa-operational-eo-missions/envisat, 2012.

Fukao, S., Hashiguchi, H., Yamamoto, M., Tsuda, T., Nakamura, T., and Yamamoto, M. K.: Equatorial Atmosphere Radar (EAR): System description and first results, Radio Sci., 3, doi:10.1029/2002RS002767, 2003.

Haslam, C. G. T., Wilson, W. E., Graham, D. A., and Hunt, G. C.: A further $408 \mathrm{MHz}$ survey of the northern sky, Astron. Astrophys. Sup., 13, 359-394, 1974.

Haslam, C. G. T., Klein, U., Salter, C. J., Stoffel, H., Wilson, W. E., Cleary, M. N., Cooke, D. J., and Thomasson, P.: A 408 MHz allsky continuum survey. I - Observations at southern declinations and for the North Polar region, Astron. Astrophys., 100-2, 209$219,1981$.

Haslam, C. G. T., Salter, C. J., Stoffel, H., and Wilson, W. E.: A $408 \mathrm{MHz}$ all-sky continuum survey. II - The atlas of contour maps, Astron. Astrophys. Sup., 47, 1-143, 1982.

Latteck, R., Singer, W., Rapp, M., Vandepeer, B., Renkwitz, T., Zecha, M., and Stober, G.: The new MST radar on Andøya: System description and first results, Radio Sci., 47, doi:10.1029/2011RS004775, 2012.

Maeda, K., Alvarez, H., Aparic, J., May, J., and Reich, P.: A 45-MHz continuum survey of the northern hemisphere, Astron. Astrophys. Sup., 140, 145-154, 1999.
Mayer, C. H. and Hollinger, J. P.: Polarized brightness distribution over Cassiopeia A, the Crab Nebula, and Cygnus A at $1.55-\mathrm{cm}$ wavelength, Astrophys. J., 151, 53-63, 1968.

de Oliveira-Costa, A., Tegmark, M., Gaensler, B. M., Jonas, J., Landecker, T. L., and Reich, P.: A Model of Diffuse Galactic Radio Emission from $10 \mathrm{MHz}$ to $100 \mathrm{GHz}$, Mon. Not. R. Astron. Soc., 338, 247-260, doi:10.1111/j.1365-2966.2008.13376.x, 2008.

Palmer, R., Vangal, S., Larsen, M. F., Fukao, S., Nakamura, T., and Yamamoto, M.: Phase calibration of VHF spatial interferometry radars using stellar sources, Radio Sci., 31, 1996.

Reich, P., Reich, W., and Fürst, E.: The Effelsberg $21 \mathrm{~cm}$ radio continuum survey of the Galactic plane between $1=95.5^{\circ}$ and $1=240^{\circ}$, Astron. Astrophys. Sup., 126, 413-435, 1997.

Renkwitz, T., Singer, W., Latteck, R., and Rapp, M.: Multi beam observations of cosmic radio noise using a VHF radar with beam forming by a Butler matrix, Adv. Radio Sci., 9, 349-357, doi:10.5194/ars-9-349-2011, 2011.

Renkwitz, T., Singer, W., Latteck, R., Stober, G., and Rapp, M.: Validation of the radiation pattern of the Middle Atmosphere Alomar Radar System (MAARSY), Adv. Radio Sci., 10, 245-253, doi:10.5194/ars-10-245-2012, 2012.

Renkwitz, T., Latteck, R., Singer, W., and Stober, G.: Validation of the receiving pattern of the MAARSY phased antenna array, Proceedings of the 13th International Workshop on technical and Scientific Aspects of MST radars, accepted, 2013.

Roger, R. S., Costain, C. H., Landecker, T. L., and Swerdlyk, C. M.: The radio emission from the Galaxy at $22 \mathrm{MHz}$, Astron. Astrophys. Sup., 137, 7-19, 1999.

Stober, G., Singer, W., and Jacobi, C.: Cosmic radio noise observations using a mid-latitude meteor radar, J. Atmos. Sol.-Terr. Phys., 73, 1069-1076, doi:10.1016/j.jastp.2010.07.018, 2011.

Swarnalingam, N., Hocking, W., and Argall, P.: Radar efficiency and the calculation of decade-long PMSE backscatter crosssection for the Resolute Bay VHF radar, Ann. Geophys., 27, 1643-1656, doi:10.5194/angeo-27-1643-2009, 2009a.

Swarnalingam, N., Hocking, W., Singer, W., and Latteck, R.: Calibrated measurements of PMSE strengths at three different locations observed with SKiYMET radars and narrow beam VHF radars, J. Atmos. Sol.-Terr. Phys., 71, 1807-1813, doi:10. 10.1016/j.jastp.2009.06.014, 2009b.

Troms $\varnothing$ Geophysical Observatory: Geomagnetic Data, magnetometer online access, http://flux.phys.uit.no/geomag.html, 2012.

Vinyajkin, E.: The Secular Decrease of the Crab Nebula at 927 and 151.5 MHz, Astrophysics, arXiv:astro-ph/0502033v1, 2005.

Zecha, M.: Untersuchung der polaren sommerlichen Mesosphäre mit unterschiedlichen Radarverfahren im VHF-Bereich, Ph.D. thesis, University Rostock, 1999. 